\title{
Application of Geographic Information Technology in the Construc- tion of Internet of things
}

\author{
Ensheng Guan \\ Shandong Zhengyuan Geophysical Information Technology Co., Ltd. Shandong Jinan 250014, China
}

\begin{abstract}
G}$ has also accelerated the emergence of the Internet of things, the current construction of the Internet of things has become a hot topic. And in the construction of the Internet of things, a very important technology is geographic information technology, which is also a good opportunity for the development of geographic information technology. Based on this, this paper first analyzes geographic information technology and its characteristics, then analyzes the origin and development of the Internet of things, and finally analyzes the application of geographic information technology in the construction of the Internet of things, so as to provide relevant people to exchange reference.

Keywords: Geographic information technology; Internet of things; Applications
\end{abstract}

Citation: Guan Ensheng, 2019. Application of Geographic Information Technology in the Construction of Internet of things. Journal of Smart Cities, 4(1): 13-15. http://doi.org/10.26789/JSC.2019.01.005

Copyright: Application of Geographic Information Technology in the Construction of Internet of things. C) 2019 Guan Ensheng. This is an Open Access article published by Urban Development Scientific Publishing Company. It is distributed under the terms of the Creative Commons Attribution-Noncommercial 4.0 International License, permitting all non-commercial use, distribution, and reproduction in any medium, provided the original work is properly cited and acknowledged.

\section{Introduction}

With the continuous development of the information age, 2020 is also the first year of $5 \mathrm{G}$ development, which has played a great role in promoting the development of the Internet. In the construction process of the Internet, geographic information technology is indispensable. Geographic information technology provides a lot of support, and the development of the Internet can also drive the development and progress of geographic information technology. These two promote each other and promote China's Internet of Things construction to reach the international leading ranks.

\section{Geographic information technology and its characteristics}

In real world, no matter where we are, we are all in a specific geographical environment, and the spatial geographical position around us is permanent. The geospatial information has its features and characteristics, which is related to each other. Geographic information technology can collect, integrate and correlate these information, so that it is presented digitally in the computer. A major feature of geographic information technology is that all geographic information is the same, and the integrated and associated information and information disassem- bly methods are also the the same level, which will not be changed due to the different purposes of users. Geographic information technology, including spatial analysis technology, data mining technology, spatial information visualization and data integration and GIS modeling. The spatial analysis technology includes six aspects, one is the acquisition and pre-processing of spatial data, the other is the transformation of attribute data and spatial scale, the third is the exploration and analysis of spatial information, the fourth is geographical statistics, the fifth is the analysis of lattice data, and the last is the prediction of complex information.

\section{Origin and development of the Internet of Things}

The concept of the Internet of Things was first proposed by the Massachusetts Institute of Technology in the United States. It is called network radio Frequency Identification system (REID). It refers to the use of radio frequency identification and other information sensing devices to connect items to the network, so that items can be intelligently managed and identified. The concept of the Internet of Things was formalized at the World Summit on the Information Society in 2005. Developed countries started earlier. As early as 2009, the United States put forward the Internet of Things as one of the important strate- 
gies to promote economic development. At the same time, in 2009, the European Union also put forward ideas on the development and management of the Internet of Things in a systematic and comprehensive way for the first time. The European Platform for Intelligent Systems Integration predicts a four-stage development of the Internet of Things (IOT), with large-scale adoption in logistics and retail around 2010, the ability to connect objects to objects by 2015 , and the ability to connect things to semi-intelligent devices by 2020. Finally, after 2020, the intelligent between objects will be fully realized.

China began to study policies to promote the development of the Internet of Things in 2006, and since then began to popularize the concept of the Internet of Things and formulate and implement policies. At present, China's domestic Internet of Things accessories design and manufacturing, short distance wireless communication, software processing system supporting facilities, system equipment manufacturing and network operation and other aspects have been able to scale into an industrial chain. At present, China's Internet of Things ecology has made great progress, is also widely spread, applied to various fields, but there are still some key nodes to be broken, I believe that with the continuous investment in the construction of the Internet of Things, will eventually be solved.

\section{Application of geographic information technology in the construction of Internet of Things}

The Internet of Things can make intelligent organic connections between people and things, and these connections are based on the application of geographic information, which is one of the important foundations for the realization of the Internet of Things.

Internet of the difficulty is how to integrate the massive real complex data sharing, in the Internet of things, compared the data in the network information data of the world is more complicated, and is the real data of real world, there are different methods of data collection, data carrier is more, and there are many fuzzy hidden data. So use in the development and change of multi-source data integration technology of multi-source data fusion technology can greatly enhance the utilization efficiency of heterogeneous data, can be in the Internet of things a lot of multi-source information comprehensive utilization, effectively avoid the repeated investment using multi-source data and deal with the problem of fuzzy data and implicit data. Spatial information visualization technology can make users have a more intuitive and clear understanding of Internet of Things services, which is convenient for the Internet of Things to serve people and provide users with fast and convenient information. For example, in the navigation system, the use of spatial visualization technology can greatly enhance people's understanding of the unfamiliar environment, so that people can more conveniently meet their own needs. Spatial analysis technology can be made in the huge amounts of data of the Internet of things accurate access to the location of the target objects in space form and distribution of information, and timely analysis of the processing, such as in the construction of wisdom can build in a city intelligent transportation system to help improve efficiency of urban traffic and the drainage of morning and evening rush hour.

GIS application modeling technology is related to the construction of corresponding application models for specific applications, and is based on lower structured information technology and practical solutions to problems in different fields ${ }^{[1]}$.

GIS modeling technology can greatly reduce the blindness in the construction of the Internet of Things, help to build an accurate Internet of things application model, facilitate the technical personnel to more intuitively understand the collected information data, construction more close to the reality, can effectively improve the efficiency of the Internet of Things construction project, avoid the waste of resources. In the massive data flow of the Internet of Things, space mining technology can be used to quickly and accurately obtain valuable information, fully mining and utilizing it, to obtain the knowledge needed by users, and serve the construction of the Internet of Things. The application of these geographic information technologies can greatly improve the ability of the Internet of Things to meet users' needs, help people establish effective connections between things and people, and provide people with better solutions to many practical problems in reality.

\section{The development prospect of geographic information in Internet of things}

China began to study policies to promote the development of the Internet of Things in 2006, and since then began to popularize the concept of the Internet of Things and formulate and implement policies. At present, China's domestic Internet of Things accessories design and manufacturing, short distance wireless communication, software processing system supporting facilities, system equipment manufacturing and network operation and other aspects have been able to scale into an industrial chain. At present, China's Internet of Things ecology has made great progress, is also widely spread, applied to various fields, but there are still some key nodes to be broken, I believe that with the continuous investment in the construction of 
the Internet of Things, will eventually be solved. In the future, geographic information will be greatly developed and utilized in the Internet of Things, and will play a great role in the construction of smart cities, intelligent transportation and other fields. In urban planning, it can help urban planners plan cities more scientifically and reasonably. In the field of intelligent transportation, the application of geographic information can help car owners to find and plan the optimal path, and can help traffic police departments to divert traffic and relieve traffic pressure. In wisdom logistics, geographic information after combined with Internet of things will bring changes to the logistics industry, also believe in the near future, with the rapid development of Internet of things, the geographic information application in the Internet of things will be more broad, the application field of more and more, to better serve the public, give full play to the advantages of geographic information in the Internet of things.

\section{Conclusion}

To sum up, geographic information has its invariability, so we can use geographic information technology to fully excavate and analyze it and find the connection between different information factors, so that we can better understand the laws behind it. Internet of things is the key to give play to the role of its need to its deployment of each sensor can have its space position, to get to the position of all information, give full play to the advantages of the geographic information technology, the use of all kinds of sensing widely deployed sensor devices, collect a large number of real data to the platform, through optimizing processing to create a platform of intelligent system, With this system, we can make a huge difference in city services, emergency response and macro decision making. In the development of the Internet of Things, its application scenarios are constantly improved and its advantages in spatial layout and planning are brought into play.

\section{References List:}

[1] Han Liang, Tan Jiqiang, Wang Jinliang. Application of Geo-information Techniques for Construction of IOT\% [J]. Geomatics and Spatial Geographic Information,2011. 